\title{
CONSULTA dE ENFERMAGEM à GESTANTE *
}

\author{
* * Osa Maria Machado de Araujo
}

RBEh/05

ARAUJO, O.M.M. - Consulta de enfermagem à gestante. - Rev. Bras. Enf.; DF, 32 : 259-270, 1979.

\section{INTRODUÇÃO}

Um dos enfoques mais atuais relacicnados com as atividades de enfermagem a nível ambulatorial è a consulta de enfermagem através da qual a/o Enfermeira/o desempenha um papel relevante junto à slientela, num sentido bem mais abrangente, objetivando uma assistência integralizada em prol da melhoria do estacio de saúde do assistido.

O aproveitamento de profíssionais nessa área de atuação representa um passo significativo da enfermagem, constuindo-se em um $2 .^{\circ}$ plano as funções gerenciais dentro dos padrōes de atribulçöes da/o Enfermeira/o nas setores ambulatoriais.

A consuita de enfermagem à gestante descrita neste trabalho vem sendo desenvolvida em uma das Unidades Médico Assistenciais co INAMPS, em Sergipe, cuja metodologia adotada baseiase no Processo de Enfermagem como forma de sistematizar os procedimentos de intervenção de Enfermagem a partir dos problemas identificados na cliente.
As etapas do processo empregadas na consulta, referem-se ao Histórico de Enfermagem, Diagnóstico de Enfermagem e Plano Assistêncial, simplificados de forma a atender de maneira funcional os objetivos que a atividade pretende atingir.

Importância da aplicabilidade do Processo na Consulta de Enfermagem:

a) propiciar uma visão global das condiçōes de saúde da gestante e das açōes de enfermagem;

b) individualizar a assistência pres. tada;

c) possibilitar o acompanhamento adequado da evolução das condiçōes da gravidez por parte do profissional;

d) fornecer dados paia futuras pesquisas na área Materno-snfantil.

\section{2 - CONCEITUAÇÃO}

Consulta de Enfermagem é definida como sendo "um conjunto de ações pres-

- Tema Livre apresentado no XXXI CBEn - Fortaleza-Ceará - 1979.

* Chefe da Equipe de Enfermagem da Secretaria Regional de Medicina Social do INAMPS - Sergipe. 
ARAUJO, O.M.M. - Consulta de enfermagem a gestante. - Rev. Bras. Enf.; DF, 32 : 259-270, 1979.

tadas pela (0) Enfermeira (o) à pessoa sadia, de forma sistemática e completa, especificameiite relacionadas com o controle da gestante de baixo risco e do crescimento e desenvolvimento da criança sadia".

\section{3 - MÉTODOS DO PROCESSO DE ENFERMAGEM}

\section{1 - Histórico de Enfermagem}

Histórico de Enfermagem é o meio utilizado para obtenção de informações a respeito da pessoa a ser assistida, permitindo o profissional conhecer, identificar e analisar as situações apresentadas, de modo a ser planejada adequaJamente a assistêncıa de enfermagem.

O levantimento de dados e a análise da situação permitem a seleção dos problemas existentes e a formulação do Diagnóstico de Enfermagem, os quais servem de base para a elaboração do Plano Assistencial.

Podemos aqui definir Problemas de Enfermagem, como sendo toda situação ou condição que requeira para a sua solução cuidacios ou intervenção do profissional de enfermagem.

O H.E. é feito por ocasião da primeira consulta, em formulário próprio, onde estão contidos dados relativos à entrevista, exame fisico e obstétrico, conforme roteiro sistematizado do formulário constante da folla n..$^{\circ} 10$.

\section{2 - Plano Assistencial de Enfermagem}

O Plano Assistencial objetiva o planejamento racional cla assistência de enfermagem a ser prestada à gestante através do atendimento de suas necessidades básicas afetadas.

A elaboração do Plano Assistencial é feita, portanto, com base nos problemas de enfermagem estabelecidos, sendo sua aplicação imediata, dada a peculiaridade do atendimento.
Podemos classificar como intervenção de enfermagem no Plano Assistencial da consulta, os critérios de Orientação e Encaminhamento, o último quando os problemas da resolução por parte de outro profissional.

O formulálio do Plano Assistencial consta da folha n. ${ }^{\circ} 11$.

\section{4 - CARACTERISTICA DO ATENDIMENTO}

Especificamente, quatro (4) são as atividades básicas desenvolvidas por ocasião da consulta e que englobam:

1 - Entrevista;

2 - Exame Físico;

3 - Exame Obstétrico;

4 - Orientação.

\section{$4.1-$ Entrevista}

Compreende-se como entrevista o arrolamento de dados relativos a:

a) identificação da gestante;

b) antecedentes pessoal e familiar;

c) condiçõos sócio-econômicas e culturais;

d) situações relacionadas com 0 estado gestacional;

e) condiçōes de saúde atual.

\section{2 - Exame Físico}

O Exame Físico compreende:

a) verificação do peso e altura;

b) TRP e PA;

c) inspeção da mama;

d) condiçōes dos dentes;

e) existência de edema e varizes. 
ARAUJO, O.M.M. - Consulta de enfermagem à gestante. - Rev. Bras. Enf.; DF, 32 : 259-270, 1979.

\section{3 - Exame Obstétrico}

De acordo com o estágio da gestação distinguimos como imprescindível, as açōes abaixo enumeradas:

a) medida da circunsferência abdominal;

b) fundo ào útero;

c) apresentação;

d) situação;

e) posição;

f) batimentos cárdio-fetal - BCF.

\subsection{Orientação}

As orientações, como já foram descritas anteriormente, estão voltadas para os problemas identificados, destacandose também, as informaçōes sobre puerpério, cuidados com a criança e medidas gerais de proteção à saúde.

\section{5 - MODALIDADE DE ATENDIMENTO}

A assistência obstétrica do INAMPS é regulamentada pela OS N.O SAM-301.1, de 18 de março de 1968 (ex-INPS), onde está definida a modalidade da assistência a ser prestada a beneficiária gestante, que compreende 0 atendimento no pré-natal, no parto e no puerpério.

No que concerne ao pré-natal, a referida os recomenda que deve ser iniciada precocemente, incluindo-se orientação à gestante.

Analisando os critérios de freqüência estabelecidos, podemos distinguir a realização de oito (8) consultas com abrangência a todo período gestacional.

Considerando a grande problemática que cnvolve o grupo materno-infantil em decorrência dos índices elevados de mortalidade e morbidade, 0 engajamento da equipe de enfermagem nessa assistência, através das crientaçōes sistematizaclas segundo as necessidades individuais identificadas, constituirá uma so- mação de esforços no sentido de ser atingida a melhoria das condiçōes de saúde do grupo enfocado. Deste modo, as açōes de enfermagem serāo prestadas através da consulta de enfermagem, segundo os critérios descritos anteriormente, podendo ser adotado o sistema intercalado entre $o$ atendimento médico e o de enfermagem tornando-se, entretanto, imprescindível que o primeiro atendimento seja realizado também pela enfermeira, pelas seguintes razōes.

a) necessidade de elaboração ào Histórico de Enfermagem, tornando-se possível um connecimento detalhado a respeito da gestante a ser assistida;

b) com base nesses dados poder efetuar o plano assistencial desde o início ảa gestação;

c) dar condiçōes de ser estabelecido o controle dos próximos comparecimentos.

Com relação ao tempo necessário para realização da consulta, verifica-se que a enfermeira dispende em média 30 minutos nos primeiras atendimentos $\mathrm{e}$ 15 minutos nos atendimentos subseqüentes.

\section{6 - RECURSOS NE9CESSARIOS}

\section{$6.1-$ Pessoal}

A atividade de consulta de enfermagem é prerrogativa da (o) Enfermeira (o), conforme consta das atribuiçōes desse profissional contiāas no Regimento Interno do Serviço de Enfermagem de Postos de Assistência Médica do INAMPS.

O Auxiliar de Enfermagem exerce atividade de apoio, tendo como atribuiçōes:
a) a pré-consulta;
b) visita domiciliar; 
ARAUJO, O.M.M. - Consulta de enfermagem à gestante. - Rev. Bras. Enf.; DF, 32 : 259-270, 1979.

c) eciucação sanitária individual;

d) registros de dados estatísticos $\mathrm{e}$ das atividades efetuadas.

O quantitativo de Enfermeiras(os) e de Auxiliar de Enfermagem, será determinado por cada serviço, levando-se em contas as metas programadas.

\section{7 - AREA FISICA E MATERIAL}

As diferentes atividades desenvolvidas nas prestação da assistência de enfermagem à gestante, englobam açōes educativas de caráter grupal e imunização, sendo portanto, indispensáveis as seguintes áreas:

01 sala para consulta de enfermagem;

01 sala para vacinação;

01 sala para reuniōes de grupo.

A especificação abaixo, relaciona-se com a compasição da sala para consulta de enfermagem:

Mesa secretária para consultório ... 1

Cadeira ....................... 2

Mesa para exame clínico ......... 1

Balança autropométrica (pesa-gente) 1

Escadinha $\ldots \ldots \ldots \ldots \ldots \ldots \ldots, 1$

Armário vitrine $\ldots \ldots \ldots \ldots \ldots \ldots, 1$

Mesinha auxiliar $\ldots \ldots \ldots \ldots \ldots \ldots, 2$

Biombo triplo ................ 1

Caixa coletora para papéis ........ 1

Ventilador $. . . \ldots \ldots \ldots \ldots \ldots \ldots, 1$

Bandeja retangular .............. 1

Aparelho de pressão arterial completo 1

Arquivo para ficha $6 \times 9$ gaveta cupla 1
Estetoscópio de Pinard $\ldots \ldots \ldots \ldots \ldots, 1$

Termômetro .................. 1

8 - COMPLEMENTAÇAO DA CONSULTA

Complementa a consulta de enfermagem as seguintes açōes:

a) Encaminhamento:

- Ao Médico

- A Nutricionista

- A Assistente Social

- Ao Odontólogo

- A Vacinação

- A outras Profissionais ou Serviços.

b) Visita Domiciliar:

- A gestante faltosa

- A puérpera.

c) Cursos de orientação Pré-Natal, segundo programação estabelecida.

\section{9 - CONCLUSĀo}

O estudo evidenciou a atuação da/o Enfermeira/o na assistência pré-natal, através da realizaçáo da consulta de enfermagem. A adoçăo de fases do Processo de Enfermagem como forma de metodotizar as açōes desenvolvidas pelo profissional, constitui um padrão de atendimento, com reflexos positivos no nível de trabalho realizado.

O preparo específico do profissional na área obstétrica e seu contínuo aperfeiçoamento técnico torna-se imprescindível à prestação eficiente da assistência de enfermagem. 
ARAUJO, OM.M. - Consulta de enfermagem à gestante. - Bet. Bire. Baf.; DF, 32 : 259-270, 1979.

MPAS - INAMPS

PROGRAMA DE SAÚDE COMUNITARIA

POSTO

HISTÓRICO DE ENFERMAGEM

1. Identificoģoo:
NOME:
EST.CIVIL:
NOME DO CONJUGE: PROFISSAO:
RESIDENCIA:

2. Entrevista e observaçõo da gestante:

Monerca: Tipo menstrual

Úlitimo menstruçoo: No de gestaçōes onteriores:

Parto normal ou patológico - Quantos? :

No de abortos No de grovidez gemelor:

No de filhos novos:

Condiçōes fisiológicos (intestino e urina) :

Hábitos - Fuma? Bebe? Cono:

Cendiçōes higiênicas:

Nóusecs: Vônitos: Vertigens:

Corrimento vaginal: 
ARAUJO, O.M.M. - Consulta de enfermagem à gestante. - Bev. Bras. Ene.; DF, 32 : 259-270, 1979.

\section{Antecedentes mórbidos:}

Fomilioress pols

$$
\text { möe: }
$$$$
\text { imōos: }
$$
pol do crianço: outros:

Pessoais: doençes anferiores Vocinas:

4. Condicöes sócio-econômicos e aulturaiss Nivel de escolaridode:

Renda fomilior mensal:

Quanfos pessoes vivem sob as expensos da fomilio:

Reside em coso próprio? Aluguel:

Tipo de residéncia: Piso:

Instalaçōos sanitärias:

Sistema de água: enconada Pogo: Rio:

Destino do lixo:

Possue luz elétrica?

Fez porte de outros progremes?

5. Exame Físico:
Peso: Estaturo: P.A.: TPR:
Mana: nódulas?

Denfes:

Edema: Vorizes: Caimbros:

Olhos: Ouvidos:

Dors

6. Excme Obstéirico:

Circunferência abdominal: Fundo de útero:

Mês de gesfoģo:

Data: Assinafura: 
ARAUJO, O.M.M. - Consulta de enfermagem à gestante. - Rev. Bras. Enf.; DF, 32 : 259-270, 1979.

INAMPS

PROGRAMA dE SAÚdE CO MUNITÁRIA

PLA.VO ASSISTENCIAL DE ENFERMAGEM

POSTO:

NOME: matríCULA

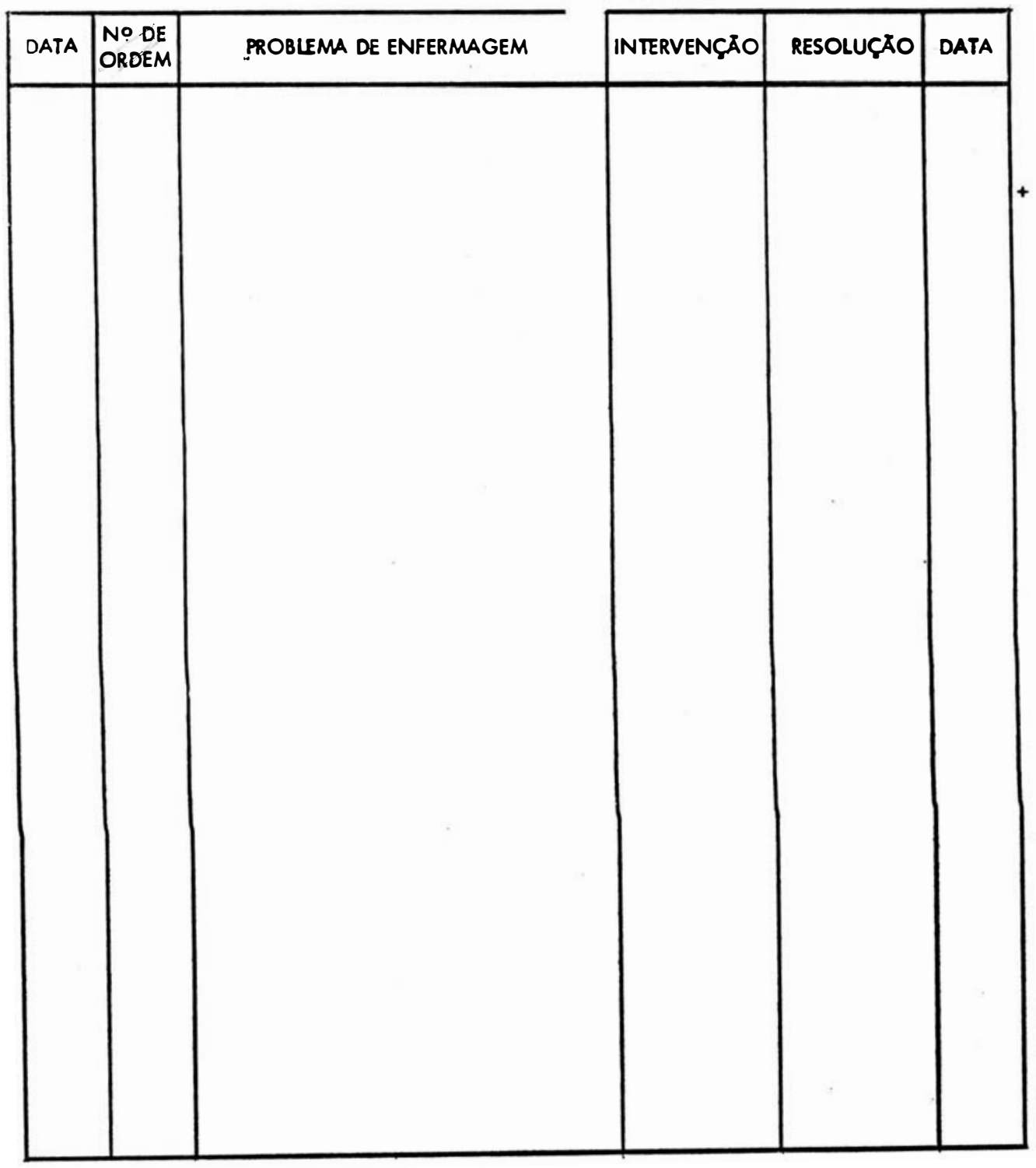

265 
ARAUJO, O.M.M. - Consulta de enfermagem à gestante. - Rev. Bras. Ene.; DF, 32 : 259-270, 1979.

INAMPS

programa de SAÚde COMUNITÁria

FICHA de aVALIAÇÃo

NOME:

MATRICULA

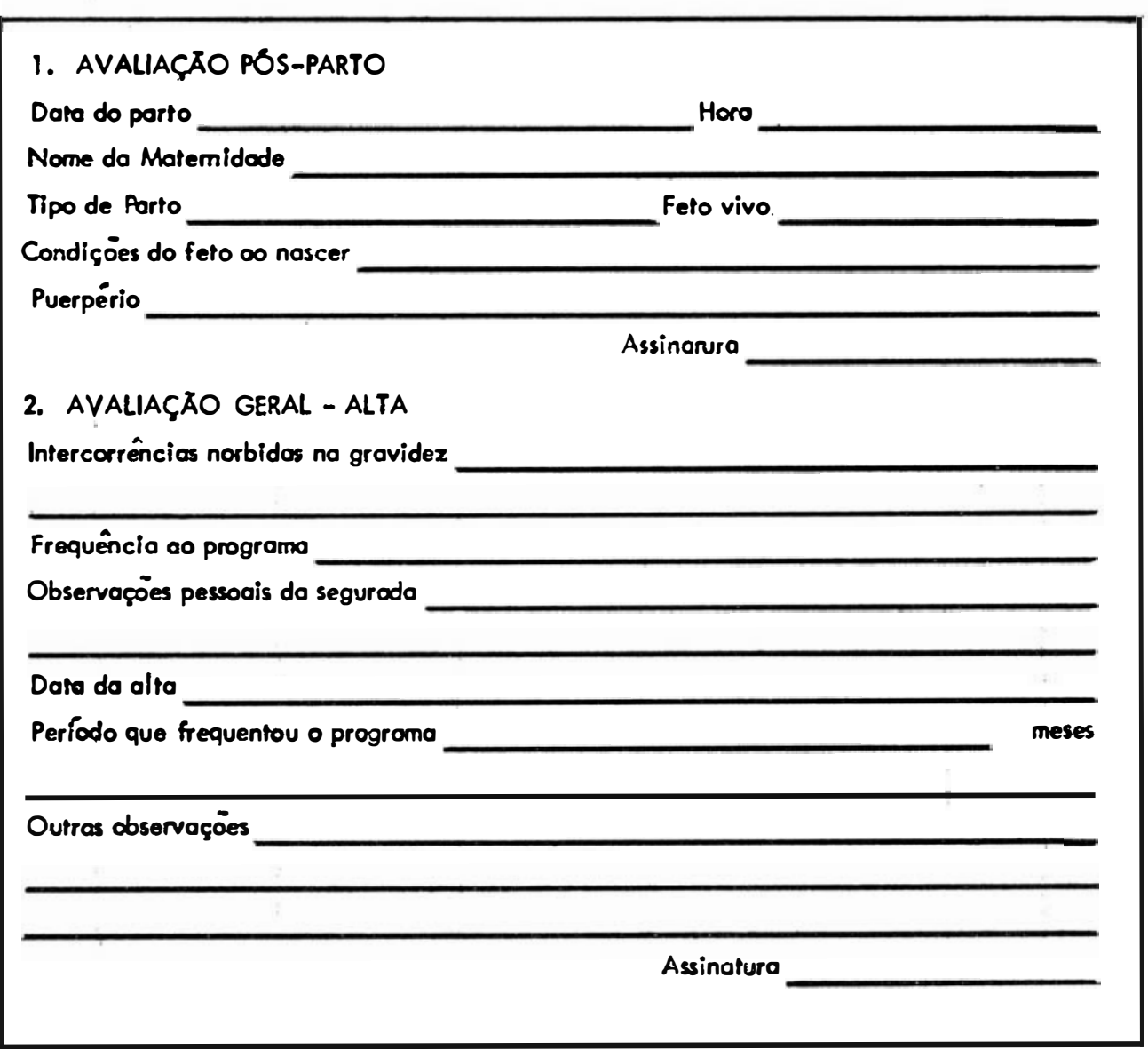


ARAUJO, O.M.M. - Consulta de enfermagem gestante. - Rev. Iran. Enf.; DF, 92 : 259-270, 1979.

INAMPS

PROGRAMA de SAÚde COMUNITÁRIA

POSTO

FICHA DE ASSISTENCIA NO PRE-NATAL

NOME MATRÍCULA

No de gestoçōes Período de Permanéncio no Progroma

Grupo Songuíneo Fotor RH

Imunizoçōo

Participoçōo em curso de Pré-Natal

Intercorrências

EQUIPE RESPONSÁVEL PELA ASSISTENNCIA

Obstetra

Enfermeira

Nutricionisto
Assistente Social

Osontólogo 
ARAUJO, O.M.M. - Consulta de enfermagem à gestante. - Rev. Bras. Enf.; DF, 32 : 259-270, 1979.

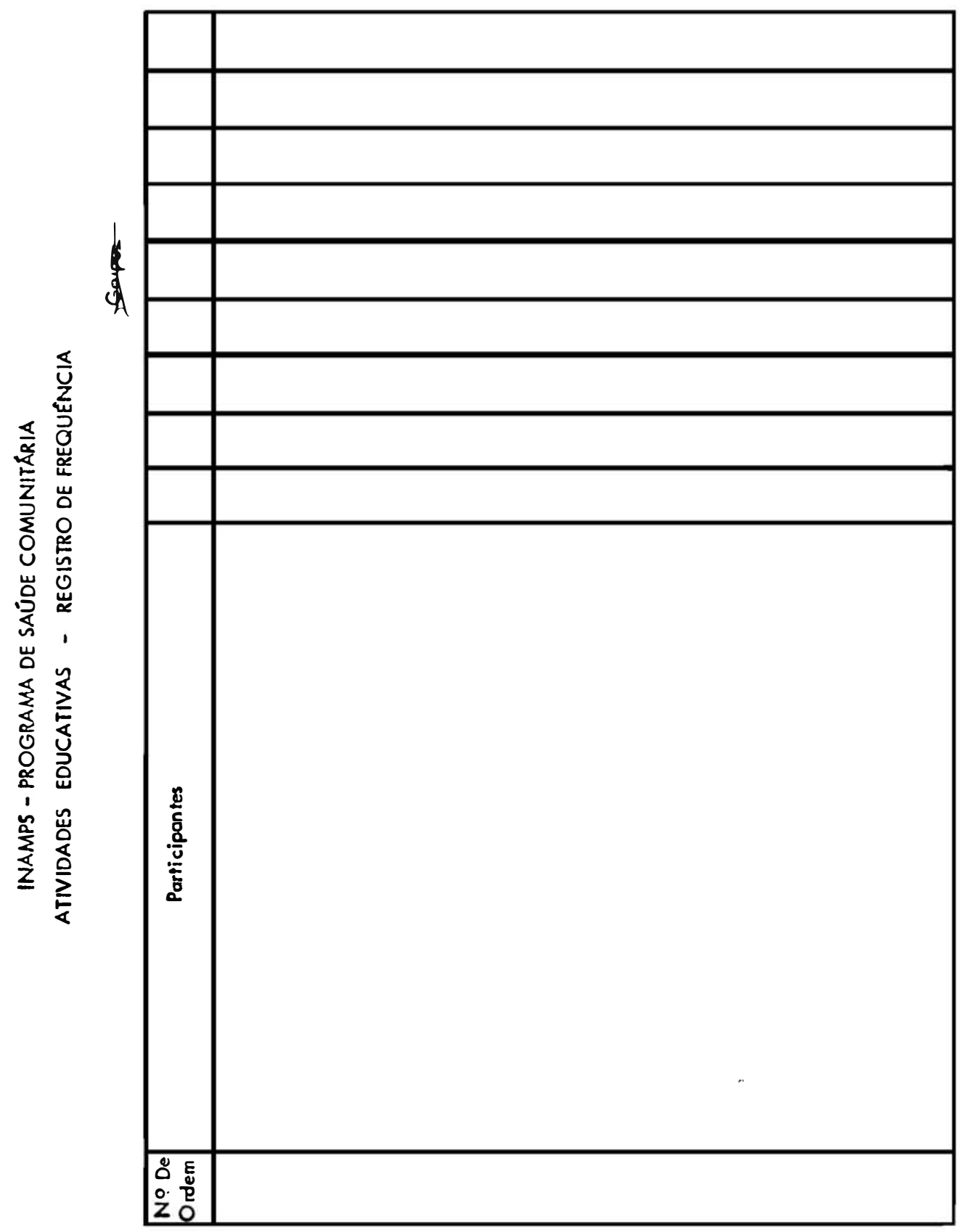


ARAUJO, OMM. - Consulta de enfermagem a gestante. - Rev. Bras. Enf.; DF, 32 : 259-270, 1979.

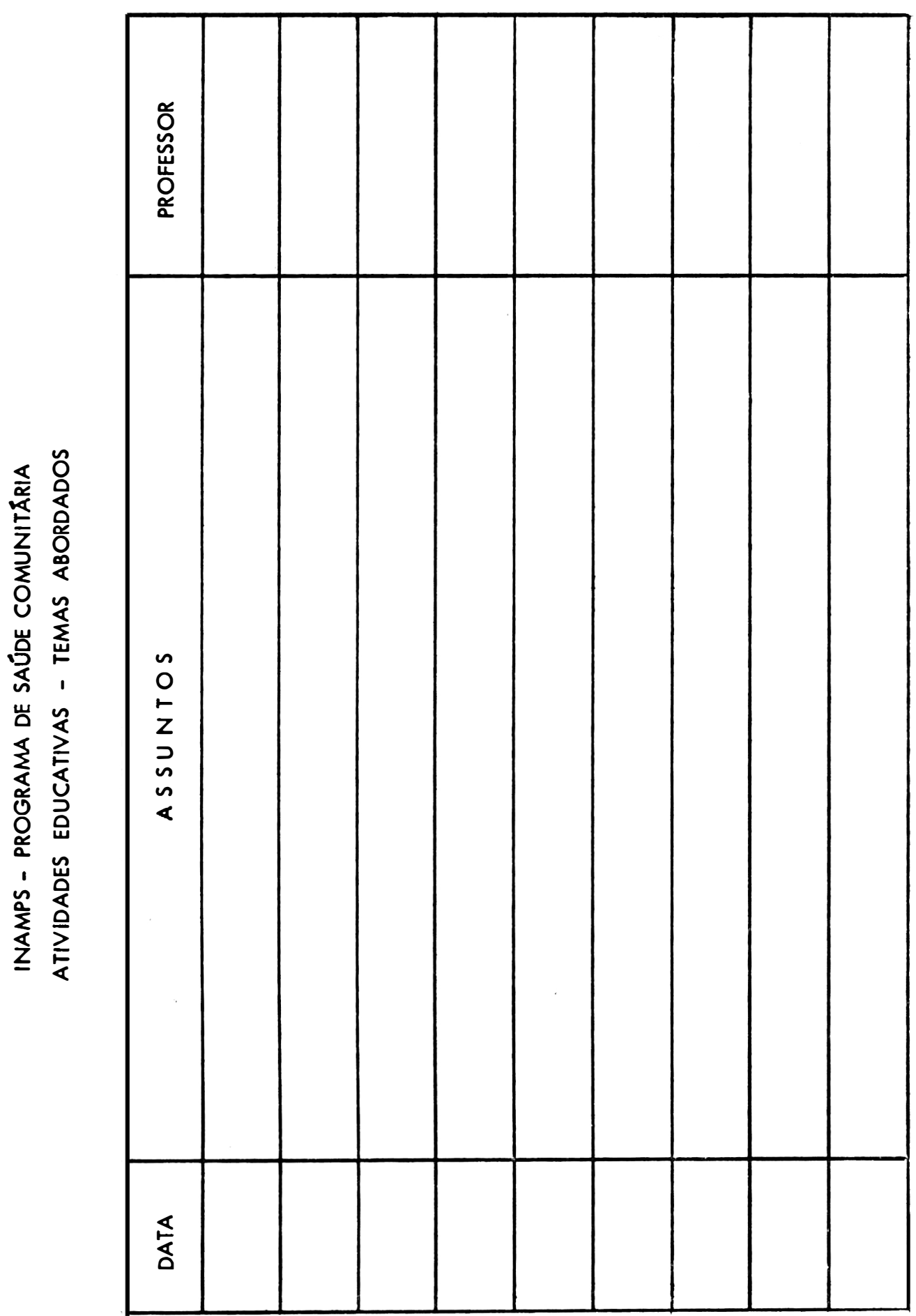


ARAUJO, O.M.M. - Consulta de enfermagem à gestante. - Ret. Bras. Enf.; DF, 32 : 259-270, 1979.

\section{BIBLIOGRAFIA}

1. CIETTO, L. - Pesquisa e desenvolvimento da enfermagem: consideraçōes, sua importância na área materno-infantil. Enf. Nov. Dimens. 2(2): 93-97, 1976 .

2. BARBOSA, M. G. F. - Aplicaçāo do Processo de Enfermagem a nivel Ambulatorial. Apostilha, Secretaria Regional de Assistência Médica. INPS. Bahia, 1977.

3. PAIM, L. - Plano Assistencial e prescrições de enfermagem. Rev. Bras. de Enf. DF. 29: 66-82, 1976.
4. HORTA, W. A. - Wanda de Aguiar A observaçāo sistematizada na Identificaçáo dos Problemas de Enfermagem em seus aspectos físicos. Rev. Bras. de Enf. 27(2) : 214-219, 1974.

5. GRELLE, F. C. - Vade-Mécum de Obstetrícia. Livraria Atheneu S.A. - Rio de Janeiro, 1963.

6. ROCH, R. M. e OKA, L. N. - Processo de Enfermagem - Avaliaçăo feita pelos alunos do departamento de enfermagem da USP. Rev. Bras. de Enf. 30: 274-285, 1977. 